\title{
The Translation Evidence Mechanism. The Compact between Researcher and Clinician
}

\author{
Janet G. Bauer* and Francesco Chiappelli
}

\begin{abstract}
UCLA School of Dentistry, Division of Restorative Dentistry, June and Paul Ehrlich Endowed Program in Geriatric Dentistry, 23-008E CHS, PO Box 951668, 10833 Le Conte Avenue, Los Angeles, California 90095-1668, USA
\end{abstract}

\begin{abstract}
Currently, best evidence is a concentrated effort by researchers. Researchers produce information and expect that clinicians will implement their advances in improving patient care. However, difficulties exist in maximizing cooperation and coordination between the producers, facilitators, and users (patients) of best evidence outcomes. The Translational Evidence Mechanism is introduced to overcome these difficulties by forming a compact between researcher, clinician and patient. With this compact, best evidence may become an integral part of private practice when uncertainties arise in patient health status, treatments, and therapies. The mechanism is composed of an organization, central database, and decision algorithm. Communication between the translational evidence organization, clinicians and patients is through the electronic chart. Through the chart, clinical inquiries are made, patient data from provider assessments and practice cost schedules are collected and encrypted (HIPAA standards), then inputted into the central database. Outputs are made within a timeframe suitable to private practice and patient flow. The output consists of a clinical practice guideline that responds to the clinical inquiry with decision, utility and cost data (based on the "average patient") for shared decisionmaking within informed consent. This shared decision-making allows for patients to "game" treatment scenarios using personal choice inputs. Accompanying the clinical practice guideline is a decision analysis that explains the optimized clinical decision. The resultant clinical decision is returned to the central database using the clinical practice guideline. The result is subsequently used to update current best evidence, indicate the need for new evidence, and analyze the changes made in best evidence implementation. When updates in knowledge occur, these are transmitted to the provider as alerts or flags through patient charts and other communication modalities.
\end{abstract}

\section{INTRODUCTION}

Advances have been made in reasoning the value and application of best evidence to patients and society. Current mechanisms include comparative effectiveness research (CER) and evidence-based research (EBR). While CER and EBD assist in reasoning individual health and treatment choices during shared-decision making with service providers, a mechanism to maximize cooperation and coordination between those who produce and those who apply evidence is lacking. The purpose of this article is to introduce the Translational Evidence Mechanism (TEM) that explains the development of data, its transformation into best evidence, clinical relevance, and meaning in practice. For healthcare, this mechanism defines the compact between researcher (research synthesis), clinician (clinical expertise, local longterm monitoring and implementation of evidence), and patients (patient choice and compliance). Thus, TEM provides the essential components of the biological, behavioral, and social interventions involved in clinical decision-making related to health care delivery.

\section{TRANSLATIONAL EVIDENCE MECHANISM}

The Translational Evidence Mechanism (Fig. 1) explains the dynamic by which best evidence is transformed into con-

*Address correspondence to this author at the UCLA School of Dentistry, Division of Restorative Dentistry, June and Paul Ehrlich Endowed Program in Geriatric Dentistry, 23-008E CHS, P.O. Box 951668, 10833 Le Conte Avenue, Los Angeles, California 90095-1668, USA; Tel: 310-825-57747; Fax: 310-641-0523; E-mail: jbauer@dent.ucla.edu sumer choices; in the case of healthcare, providing needed analyses of differing health care and service options for patients in deciding the best clinical decision. The mechanism consists of 3 components: Translational Evidence Organization, central database, and decision-making algorithm. The outcome of this mechanism is to provide end-users: patients or clients, with current, best evidence to make informed and optimal decisions regarding life choices, choices specific to any discipline in which consumer-based wants, needs, and desires are expressed. The product of such a system is typically called a clinical practice guideline (CPG). A CPG takes into account the probability (or odds ratio) of an event or events, the human preferences (expressed as values) attributed to these events, and their costs (benefits or trade-offs).

\section{TRANSLATIONAL EVIDENCE ORGANIZATION}

The Translational Evidence Organization develops, verifies, maintains, and updates current, best evidence for service providers in their consultations with patients. Initially, inputs to the organization are made in the form of an inquiry. This inquiry results from uncertainties that may arise in consumer decision-making. The inquiry drives this organization to provide in real-time, effective and efficient decision-making; in other words, in a manner that demands current best evidence for immediate decision-making in determining the optimal cost/benefit choice for the consumer. If best evidence does not exist currently, the organization initiates an internal inquiry to develop new evidence based on consumer-based wants, needs, and desires. 


\section{Transforming Scientific Evidence into Better Health Care}

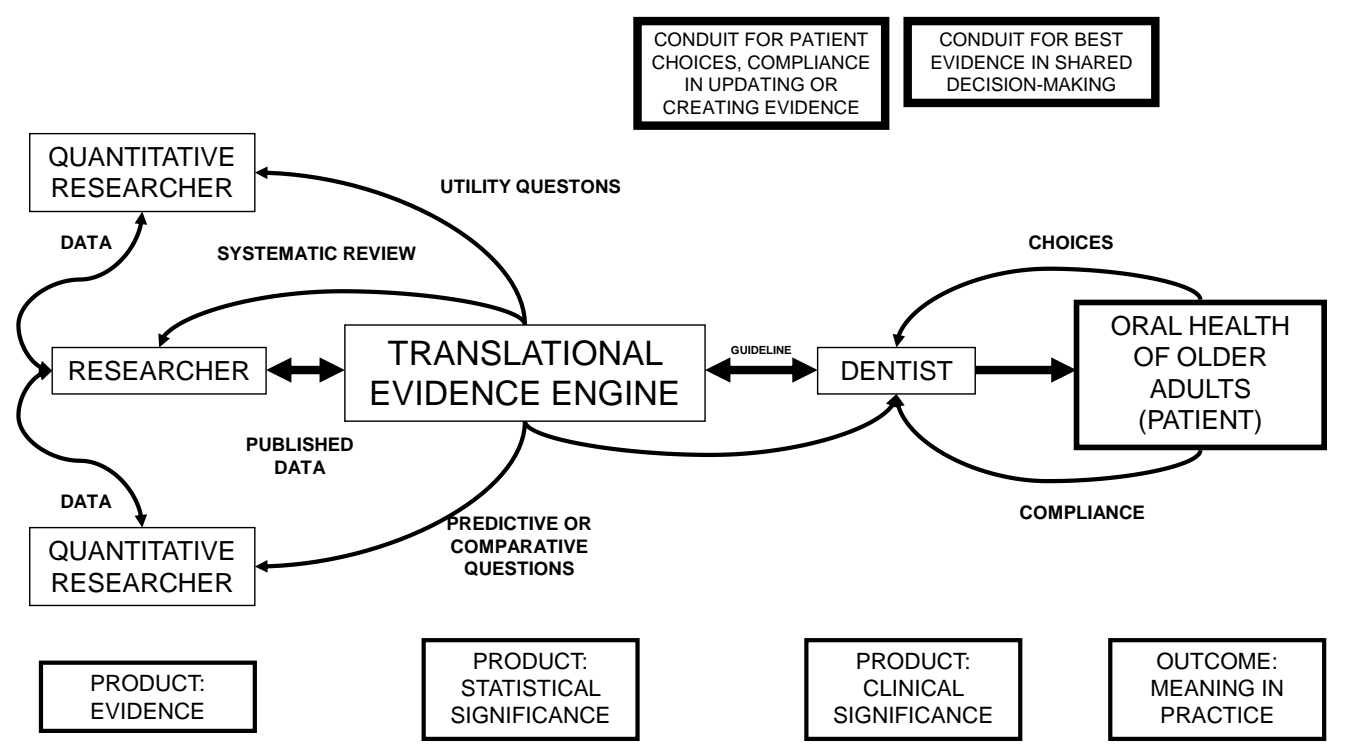

Fig. (1). Translational Evidence Mechanism.

The primary purpose of the Translational Evidence Organization is to arbitrate published evidence and, in its absence, identify research that needs to be conducted by organizational affiliates or the research community in producing needed data. The Comparative and Translational Researcher (Translational Researcher) coordinates with IT Research and Development administrators to vest a central database with evidence. Resultant research data is then defined by templates when inputted into a central database. Similarly, best evidence is defined by templates of outputs. Both inputs and outputs may be stratified within the central database by geographic, discipline, population, and/or other categorical needs. Outputs consist of arbitrated decision data produced by quantitative researchers, utility data (attitudes, beliefs, and preferences of both providers and consumers) produced by qualitative researchers, and cost data. Arbitrated best evidence meets the rigorous standards of statistical significance, the soundness and generalization of information or whether findings can be applied to similar patients in similar settings. Outputs are stated in terms of validity and reliability of study design to express confidence in providing best evidence. All are displayed in clinical practice guidelines. The CPG is the vehicle, or professional standard, that manages data for use in private practice.

Additional post arbitrators of best evidence include the provider (clinician) and consumer (patient). The clinician is concerned with clinical significance; whether research findings can be applied to individual patients. Dental clinicians, dentists, make judgments that may weight best evidence differently from researchers. Initially, clinical significance of decision data is coordinated with dentists in validating clinically national, regional, or local relevant best evidence. The dentist's evaluation of clinical significance is used by the translational researcher to reject or modify the clinical practice guideline or to re-identify and conduct investigations that produce other clinically relevant data. Personal and professional experiences, values and preferences, and appropriate practices, as well as patient well-being, quality of life issues, and costs weigh heavily on whether best evidence is used in clinical decisions. Thus, dentists are able to predict clinical outcomes in weighing risks against benefits and costs for individual patients. Dentists may also make relevant standards of care in their local practice and for specific patient population characteristics. These clinical validations are necessary to translate research data into clinically useful data for patient care.

Clinical significance also addresses the importance of the evidence that takes into consideration the long-term multifaceted monitoring of evidence in the context of human behavior (patients). However, clinical significance may vary between dentists and between patients. This difference results because dentists, as well as patients, make judgments that weigh differently personal and professional experiences, values and preferences, and appropriate practices. In other words, judgments of risk and benefits vary because of differences in weights given to these variables, especially when costs are included in the decision-making. All is important for patients in accepting best evidence in their acquiring the highest level of cost effective services, either through feefor-service or as a defined benefit of their dental insurance plan. Thus, patients become the "conceptual subject" to which best evidence is applied and quantitative and qualitative outcomes are measured. However, patients may be advocates or adversaries of evidence. Patients may also exert demands on evidence to meet specific, personal needs. They may exert influence on the development and application of knowledge that does not necessarily meet acceptance criteria of researchers and dentists, but serves a personal need. They may also exert pressure to deny the development and application of knowledge that is contrary to their philosophical 

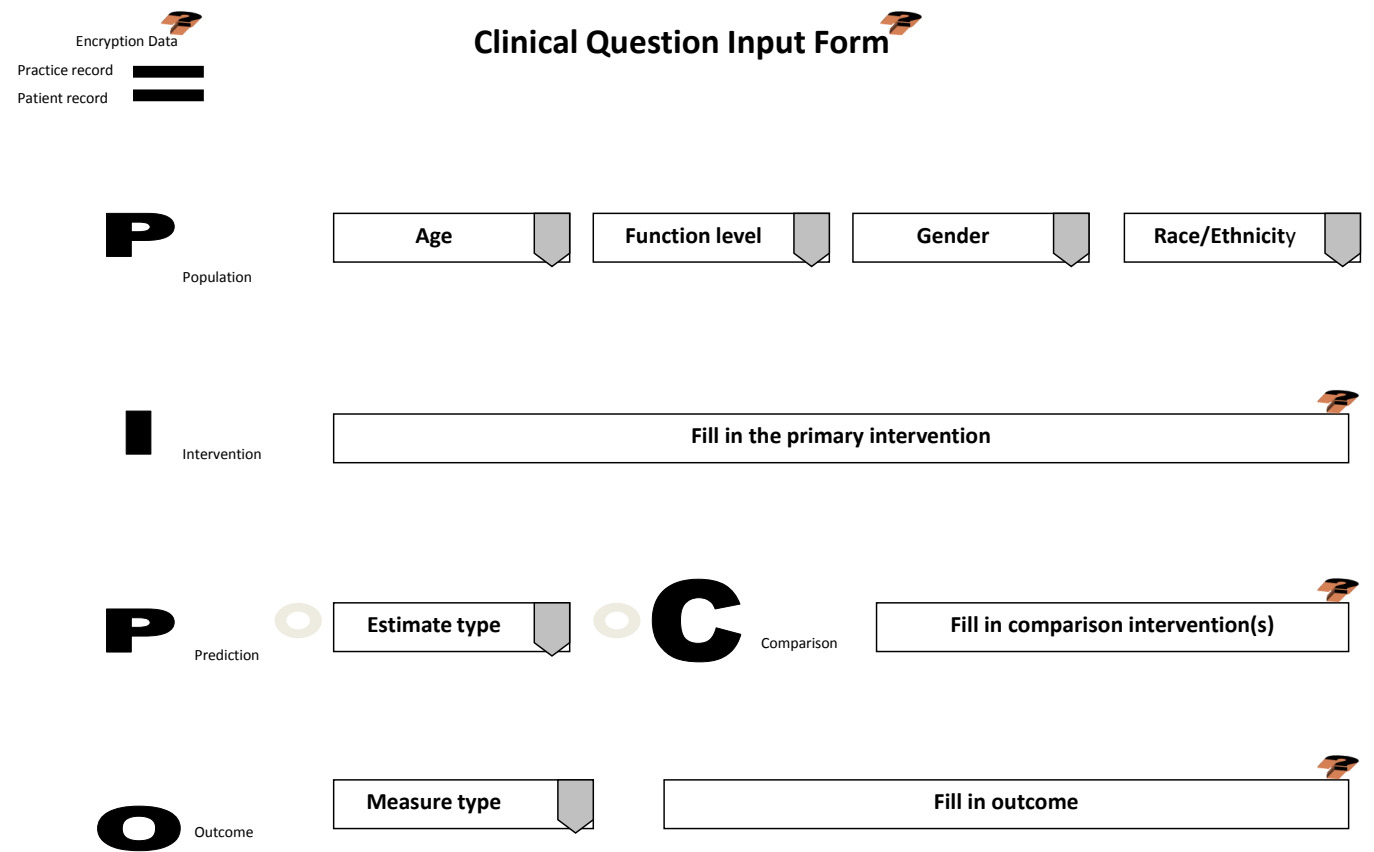

Customize Clinical Practice Guideline

Fig. (2). Clinical Question Input Template.

beliefs. Even in the profession's best efforts of informing patients with best evidence and using clinician expertise to communicate individualized, effective treatments, patients ultimately decide if treatment regimens are adhered to or rejected outright. Patient adherence, modification, or rejection of best evidence in treatment scenarios provides the translational researcher with its meaning in practice. The translational researcher uses these evaluations in updating clinically relevant data. These updates are processed using decision, Bayesian, and sensitivity analyses within the central database.

Thus, the translational researcher is the final arbiter of evidence. The translational researcher functions, simplistically, to translate basic research language into the language of the clinician and patient. In other words, basic research is assessed, evaluated, and disseminated to the clinician in a means usable for shared decision-making. The product of evidence-based dentistry is the published CPG. While there is no requirement that the published clinical practice guideline be implemented in private practice, dentists could use best evidence to offer their patients best practices in a stateof-the-art organization and facility. This requires that dentists review and effect personal, behavioral changes to accomplish care and service advances.

\section{CENTRAL DATABASE}

The central database is the repository of evidence, organized and stored utilizing evidence-based research knowledge management software (EBRsoftware). EBRsoftware [1] is maintained by information technologists and integrated within the electronic patient chart. Both health information technology and the Internet, as a vehicle for information technology, has become the most cost effective mode of information transfer $[2,3]$. It is this inclusive and reciprocal participation of all decision makers that accomplishes improvements in clinical care. The structure of the central database consists of a primary network. This primary network contains all vested evidence. The network may then be partitioned into subordinate databases. Information is then filtered and segregated per subspecialty information. Subspecialty information is that best evidence used in specific populations, geographic locales: national, regional, specialty, and local secondary networks, specialty procedural treatments, quality assurance, costs, and care delivery including facility or equipment improvements. These subspecialty databases may facilitate access based on frequency of usage.

\section{INITIATING THE MECHANISM}

During the examination appointment (initial or recall), the dentist interviews and assess the patient for $\mathrm{h} / \mathrm{her}$ health and oral status. The information is inputted into the electronic chart. Once assessments have been completed and the evaluation process of needed services or maintenance regimens begins, the dentist discusses with the patient treatment options in arriving at a treatment plan. If presenting conditions, patient characteristics, and treatment outcomes are uncertain in their benefits and harms, then the dentist participates with the patient in shared decision-making to provide informed consent, assisting the patient in making the most optimal clinical decision that meets their needs and choice in accomplishing oral health care and wellness. Using EBRsoftware, the dentist accesses a clinical input form through the electronic chart to assist in forming a clinical question regarding the treatment options and their outcomes. The input template is based on the PICO question, a standardized format for initiating evidence-based research. The PICO input form (Fig. 2) is completed by the dentist based on patient responses. 


\section{Clinical Practice Guideline}

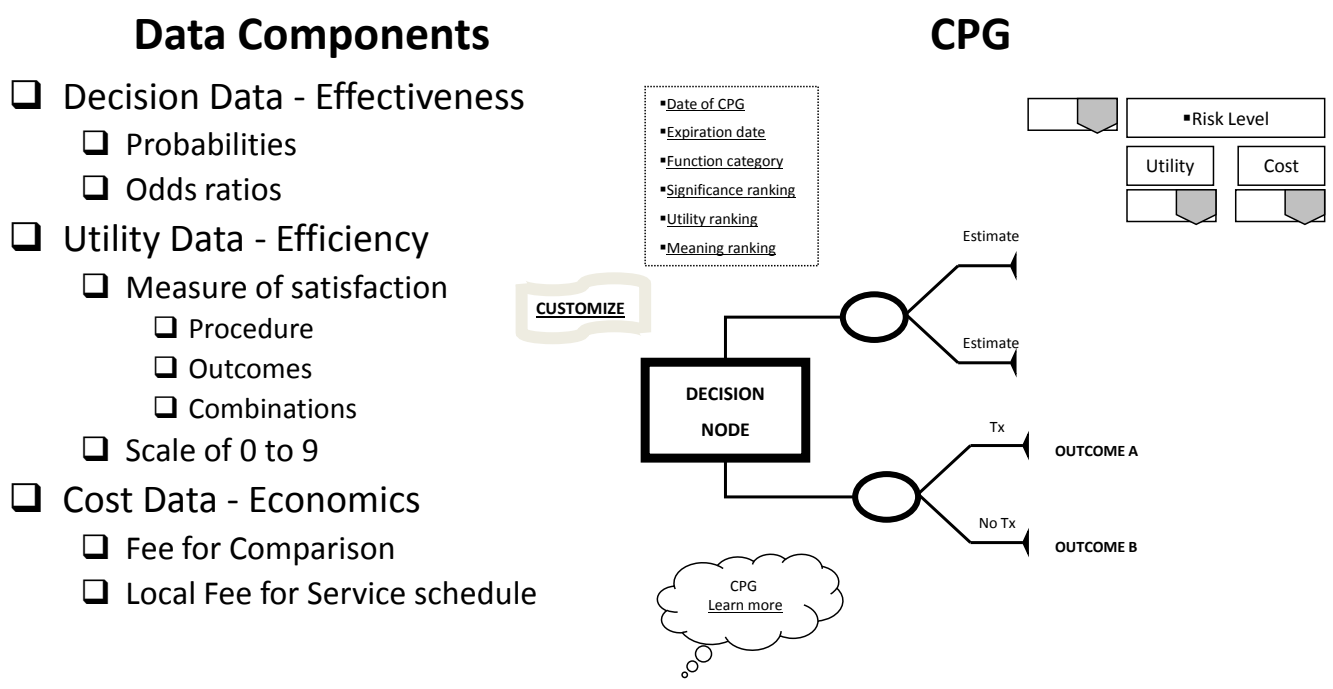

Fig. (3). Clinical Practice Guideline.

When submitted, the clinical input form will interact with the central database. Because this form is being sent through the electronic patient chart, patient histories, assessments, evaluations, and practice cost schedules are collected and all information is encrypted prior to integrating with the central database. This encryption uses technologies to insure security, privacy, and uphold the requirements of HIPAA.

Based on the query, the EBRsoftware manages relevant data to return decision and utility best evidence coordinated with practice cost schedules in a clinical practice guideline template (Fig. 3). The CPG is returned dated and specified as to expiration date of the evidence provided, the functional status (functionally independent, frail, or functionally dependent) of the patient to which the evidence applies, and, if available, the significance (statistical and clinical), utility ranking, and meaning in practice of the evidence. Other visual aids include links to "customize," changing the components of the CPG to accommodate different choices or correct mistakes, and "Learn more," to exhibit the documentation that produced the CPG and its relevant bibliography. The returned CPG is interactive in that it provides stratified risk scenarios for high, equipoise, or low risk takers that may be accessed and "gamed" through a drop down menu. Basically, the CPG provides a minimum of two treatment scenarios: treatment and no treatment with their respective outcome (best evidence) probabilities; if more options or comparisons of treatments have been requested through the clinical input form, then those will be displayed in their entirety. Along with this decision data, utilities of each outcome are provided. These utilities are based on a scale from 0 to 9 , a standard scale used in utility research.

With the patient's involvement, returned quantitative and qualitative best evidence is organized into decision, utility, and cost data for each possible option/outcome to assist in shared decision-making. If utility rankings, based on the "average patient," are not acceptable to the patient, these may be changed through drop down menus, reflecting personal preferences, values, and beliefs regarding the presented outcomes. Thus, the patient may change, or "game," rankings to compare and test different treatment scenarios in optimizing the clinical decision; or in other words, provide assessments to compare personal trade-offs between options with the "average patient." If so desired, the dentist may manipulate outcome reimbursements to provide different cost outcomes. All changes are immediately recalculated to demonstrate different treatment scenarios for enhanced informed consents. The objective of patient "gaming" is to optimize the clinical decision. Accompanying the CPG exhibit is the decision analysis statement. This statement provides a written interpretation or meaning of the treatment scenarios and their calculations (Fig. 4a, b, and c).

The decision analysis of the CPG is done by: firstly, interpreting for the patient the probability of the outcome; secondly, multiplying utility data by the probability of the outcome in interpreting the service benefit to the patient; and thirdly, multiplying the probability of the outcome by the treatment cost in determining the economic choice difference between outcomes (not applicable in Fig. 5). With these analyses, the patient can determine $h$ /hers optimal treatment option. The example decision analysis in Fig. (5) may appear absurd as a stand-alone decision. However on reflection, the information may be helpful in determining a patient's access to dental services. The resultant patient analysis and optimal clinical decision is returned to the central database to be retrieved by translational researchers in developing new research questions or modifying existing evidence. Having an efficient decision-making process has an additional benefit of involving private practice as a unit in clinical research without disrupting normal patient flow or care. Conversely, clinicians may compare local based practice norms, patient characteristics, and standards to those derived on a regional and national basis. 


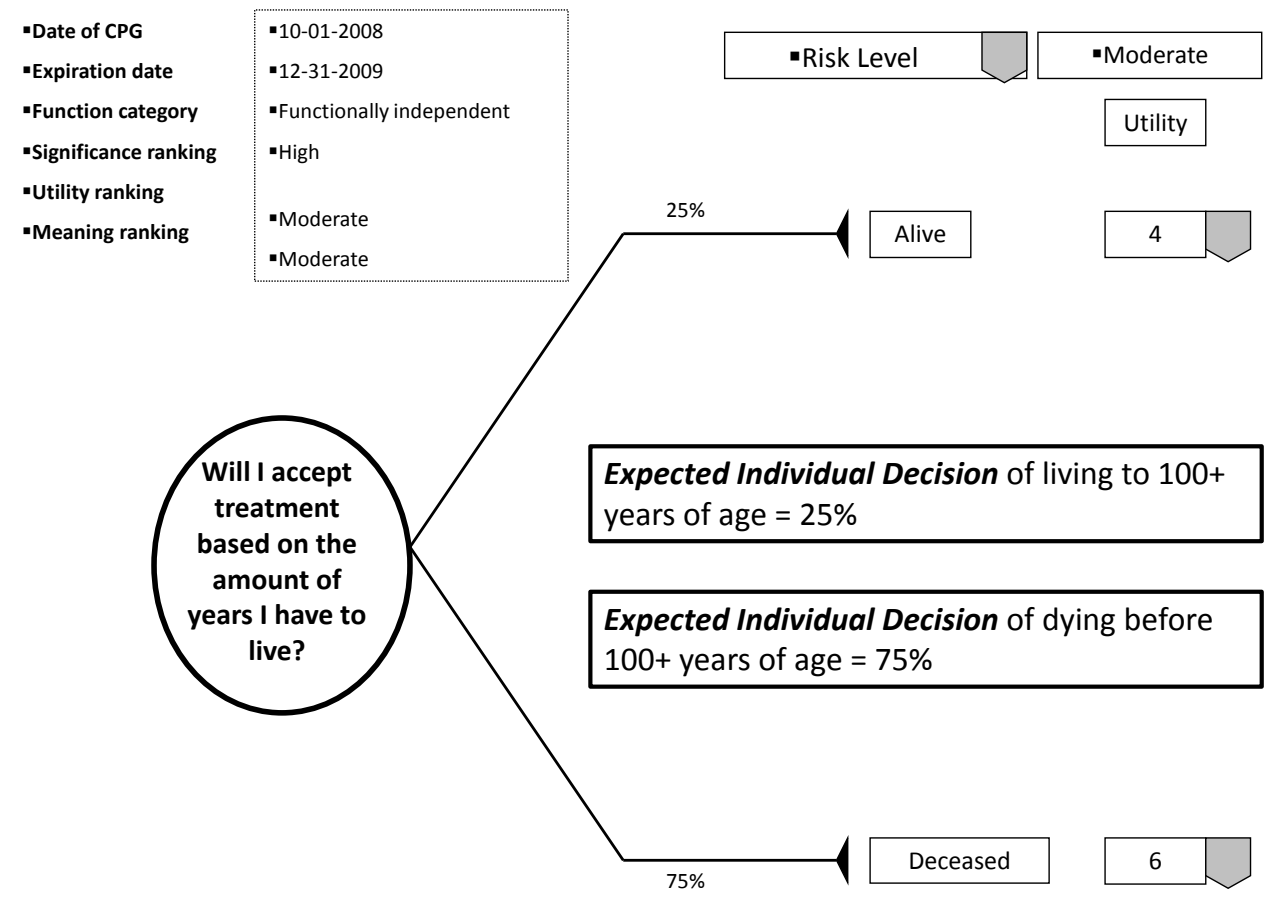

Fig. (4a). Example of decision analysis using decision data.

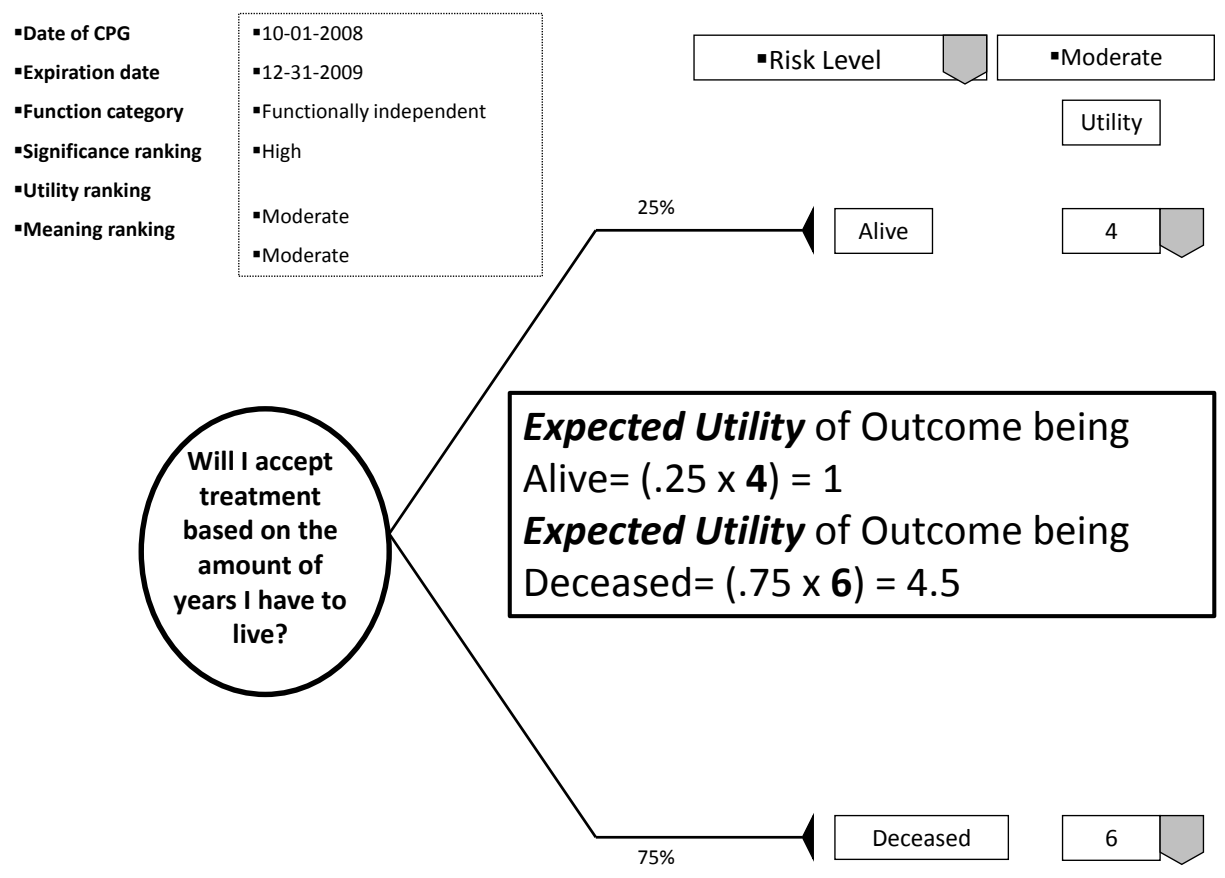

Fig. (4b). Example of decision analysis using utility data.

Once processed, the results may be transferred to the central repository, or central database. Within the central database, probabilities are updated using Bayesian analyses. Sensitivity analysis determines which components of the CPG have the greatest impact on the clinical decision. This analysis may also determine the effects of changes in one of the components or two or more of the components of the CPG. Updates may then be sent back to the dentist in learning how patient decisions differ from the "average patient" in the practice locale or change over time. This reciprocation of evidence is done through the electronic chart. It is facilitated with alerts or flags to disseminate improvements in provider understanding of the patient, $\mathrm{h} / \mathrm{hers}$ treatments, and care delivery.

\section{MEANING IN PRACTICE OF THE EVIDENCE}

Quantifying and qualifying the meaning of evidence begins at follow-up assessment visits, periodic dental examinations. For these assessment visits, the patient provides updates to decision data through patient compliance and out- 


DECISION ANALYSIS FOR PATIENT-MODERATE RISK TAKER
Meaning of Analysis
Determining the Optimal Clinical Decision
From the analysis, best evidence regarding the decision to be made is presented. The data is
combined in a way that the value of the decision may be shown. The context of the decision is
presented with respect to like decision or trade-offs made by others in the patient's average age
cohort. Using this comparison context, the patient may make h/her own Optimal Clinical
Decision. For the patient, then:
While the probability of living to $100+$ years of age is $25 \%$, the benefit of that life expectancy is 1
out of a scale of 0 to 9 .
While the probability of being deceased before reaching $100+$ years of age is $75 \%$, the benefit of
dying sooner is 4.5 .
Thus, I the patient can see that while I have a fair chance of living to $100+$ years of age, the
quality of life of those additional years is minimal. So, while not pleasant to consider, I would be
better off if I died sooner than later. Am I comfortable with that decision or do I want to differ
from my average age cohort decision?

Fig. (4c). Example of decision analysis interpreting the CPG using decision and utility data.

\section{Hypotaxis of Decision-making}

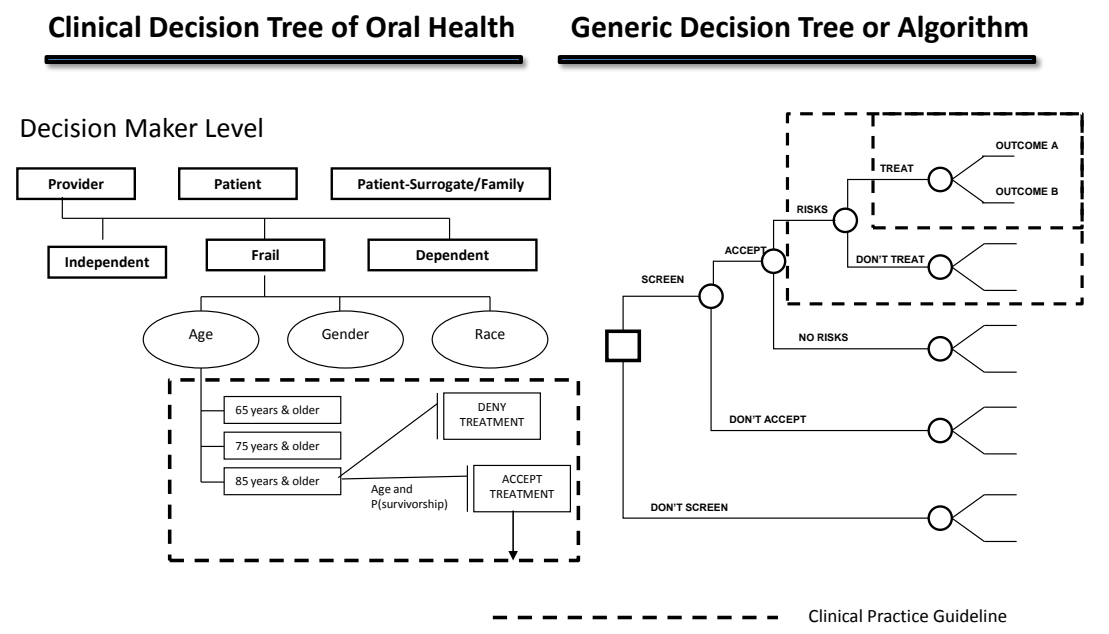

Fig. (5). Hypotaxis of decision-making.

comes dependent on healthy lifestyles and wellness. Utility data is updated by patient preferences and value of the services rendered or recommended maintenance regimens. With input from the dentist, the translational researcher uses these periodic assessments to update the CPG in identifying new areas of research or improving its usefulness in private practice through sensitivity analyses. Thus, the dentist acts as a conduit for the researcher in providing local data regarding patient choices, compliance, and treatment outcomes in updating or producing new evidence.

\section{DECISION-MAKING ALGORITHM}

A decision-making algorithm assists decision-makers in managing a decision process. Similar to evidence-based re- search, an algorithm provides a systematic understanding of decision strategy management. The basic structure of the algorithm is the decision tree. A decision tree is a visual representation of instructions to approach and manage a complex decision process. For healthcare algorithms, clinical practice guidelines become decision tree termini. An example of a healthcare algorithm for older adults is the Clinical Decision Tree of Oral Health (Fig. 5) [4].

The Clinical Decision Tree of Oral Health is used to assist decision makers in making effective and efficacious decisions regarding treatment options that best optimize treatment selection, personal utilities, and goals in improving oral health. Likewise, a decision-making algorithm structures the 
organization of evidence in the central database registry. It is by the decision-making algorithm that evidence is managed and stored. The structure of the algorithm is essential to retrieval of best evidence when an inquiry is received. It is this structure that constitutes the ordered progression of human decision-making concerning outcomes, their comparisons, and associated information to make inferences regarding preferences (values) and affected costs (benefits, or tradeoffs).

\section{SUMMMARY}

The Translational Evidence Mechanism is a compact between researcher, clinician, and patient in understanding best evidence as it applies to private practice. Since best evidence is used in informed consents regarding optimal treatment decisions, it must stand to scientific rigor, be clinically relevant, and produce changes in patient behavior. Thus, best evidence requires 3 components for effective production, validation, administration, and continuous monitoring of new knowledge and technology. The Translational Evidence Mechanism accomplishes this using a central database and decision-making algorithm. Communication of best evidence from translational researcher to clinician and patient is through the electronic chart. During shared decision-making, patients are active participants in determining the inputs of their personal life experiences and preferences directly into decision-making. Clinicians act as skilled assessors of patient presenting conditions, facilitators in patients arriving at informed and individualized decisions for personal care that meets their lifestyles and behaviors. Both act as reciprocators in a process that updates and screens best evidence for its practical implementation in patient care and meaning to societal health agendas. In return, changes in best evidence is disseminated as alerts or flags in patient charts. Thus, the Translational Evidence Mechanism uses evidence-based research methodologies in optimizing interdisciplinary resources for promoting oral health and preventing disease in individual patients by integrating best evidence, clinical experience and expertise, and patients in clinical decisionmaking, best evidence validation, and practical implementation.

\section{REFERENCES}

[1] Process of evidence-based dental decision-making, US patent application No. 60/757,647, For: methods of producing evidencebased decision, Filed 2006, UC Case No.: 2006-296, File No. (Townsend and Townsend and Crew): 02307K-16300US.

[2] Bakken S, McArthur J. Evidence-based nursing practice: a call to action for nursing informatics. J Am Med Inform Assoc 2001; 8: 289-90.

[3] Integrated healthcare association. Pay for performance (P4P) 2008 Results Report. August 2009. http://www.iha.org/p4pyr6/02\%20\%20P4P\%202008\%20Results_Report.pdf. [Accessed August 29, 2009].

[4] Bauer JG, Spackman S. Clinical decision making tree for older dental patients. In: Chiappelli F, Ed. A manual of evidence-based research for health sciences: Relevance to dentistry. New York: Nova Publishers 2007; Chapter 13. 